\title{
Last issue of journal of chemical biology
}

\author{
Rudiger Woscholski $^{1} \cdot$ Banafshe Larijani $^{2}$
}

Received: 10 September 2017 / Accepted: 11 September 2017 /Published online: 7 October 2017

(C) Springer-Verlag GmbH Germany 2017

When we founded this journal back in 2008, chemical biology was still an emerging discipline that had not yet made an impact in the publishing landscape with only a few journals and many of the other journals keeping close to their core disciplines. However, nearly a decade onwards, most journals welcome multidisciplinary manuscripts, although only a few "chemical biology" focused journals exist. Journal of Chemical Biology belongs to the latter category, publishing multidisciplinary manuscripts that cover a wide range of topics with a particular emphasis on the innovation within the physical sciences, but also informing the chemical biology community through its bulletin of interesting conferences, books, and news. Since its inception, over 150 manuscripts have been published in the Journal of Chemical Biology attracting over 1400 citations (without self-citations; web of science search on all databases) demonstrating the high impact of the work published.

However, the developments in the publishing landscape made it clear that the interests of our authors are best served by aligning ourselves with the ChemCentral Journal (https://ccj.springeropen.com), an open access publication by Springer that is listed in many indexing services including the Science Citation Index (current Impact factor: 2.442). The Journal of Chemical Biology will cease publishing, with this issue being the last one, but will continue to serve the chemical biology community in the newly formed chemical biology section of the ChemCentral Journal. In order to ensure a smooth transition and translation of the chemical biology culture and spirit, we will serve as editors for this section and would invite all potential authors to consider submitting their manuscripts to ChemCentral Journal/chemical biology section from now on.

We wish to thank the chemical biology community for all contributions including items for the bulletin. In particular, we would like to thank our bulletin editor Colin Rosser, who managed that part of our journal, as well as all our current and past editors, authors, and referees, who all made sure that we published high-quality manuscripts over the years.

Banafshe Larijani and Rudiger Woscholski

Editors-in-Chief
Rudiger Woscholski

r.woscholski@imperial.ac.uk; http://www.bio.ic.ac.uk/research/

woscholski/woscholski.html; http://www.chemicalbiology.ac.uk/

Banafshe Larijani

banafshe.larijani@ikerbasque.org; http://www.ikerbasque.net/ banafshe.larijani

1 Department of Chemistry, Faculty of Natural Sciences and Institute of Chemical Biology (ICB), Imperial College London, South Kensington campus, London SW7 2AZ, UK

2 Cell Biophysics Laboratory, Ikerbasque Basque Foundation for Science, Research Centre for Experimental Marine Biology and Biotechnology (PiE) \& Biofísika Instituto (UPV/EHU, CSIC), University of the Basque Country, Bilbao, Spain 